\title{
Sampling Correctors
}

\author{
[Extended Abstract] ${ }^{*}$
}

\author{
Clement L. Canonne ${ }^{\dagger}$ \\ Columbia University \\ ccanonne@cs.columbia.edu
}

\author{
Themis Gouleakis $\ddagger$ \\ CSAIL, MIT \\ tgoule@mit.edu
}

\author{
Ronitt Rubinfeld ${ }^{\S}$ \\ CSAIL, MIT and the Blavatnik \\ School of Computer Science, \\ Tel Aviv University \\ ronitt@csail.mit.edu
}

\begin{abstract}
In many situations, sample data is obtained from a noisy or imperfect source. In order to address such corruptions, this paper introduces the concept of a sampling corrector. Such algorithms use structure that the distribution is purported to have, in order to allow one to make "on-the-fly" corrections to samples drawn from probability distributions. These algorithms then act as filters between the noisy data and the end user.

We show connections between sampling correctors, distribution learning algorithms, and distribution property testing algorithms. We show that these connections can be utilized to expand the applicability of known distribution learning and property testing algorithms as well as to achieve improved algorithms for those tasks. As a first step, we show how to design sampling correctors using proper learning algorithms. We then focus on the question of whether algorithms for sampling correctors can be more efficient in terms of sample complexity than learning algorithms for the analogous families of distributions. When correcting monotonicity, we show that this is indeed the case when also granted query access to the cumulative distribution function. We also obtain sampling correctors for monotonicity without this stronger type of access, provided that the distribution be originally very close to monotone (namely, at a distance $O\left(1 / \log ^{2} n\right)$ ). In addition to that, we consider a restricted error model that aims at capturing "missing data" corruptions. In this model, we show that distributions that are close to monotone
\end{abstract}

*A full version of this paper is available at http://arxiv.org/abs/1504.06544

${ }^{\dagger}$ Research supported in part by NSF CCF-1115703 and NSF CCF-1319788. Some of this work was done when the author was an intern at Microsoft Research New England.

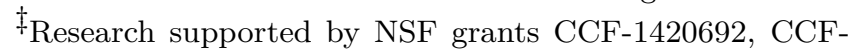
1217423 and CCF-1065125.

${ }^{{ }^{2}}$ Research supported by ISF grant $1536 / 14$ and NSF grants CCF-1420692, CCF-1217423 and CCF-1065125.

Permission to make digital or hard copies of all or part of this work for personal or classroom use is granted without fee provided that copies are not made or distributed for profit or commercial advantage and that copies bear this notice and the full citation on the first page. Copyrights for components of this work owned by others than the author(s) must be honored. Abstracting with credit is permitted. To copy otherwise, or republish, to post on servers or to redistribute to lists, requires prior specific permission and/or a fee. Request permissions from Permissions@ acm.org.

ITCS'16, January 14-16, 2016, Cambridge, MA, USA.

Copyright is held by the owner/author(s). Publication rights licensed to ACM. ACM 978-1-4503-4057-1/16/01 ...\$15.00.

DOI: http://dx.doi.org/10.1145/2840728.2840729 . have sampling correctors that are significantly more efficient than achievable by the learning approach. We then consider the question of whether an additional source of independent random bits is required by sampling correctors to implement the correction process. We show that for correcting close-touniform distributions and close-to-monotone distributions, no additional source of random bits is required, as the samples from the input source itself can be used to produce this randomness.

\section{Categories and Subject Descriptors}

G.3 [Probability and Statistics]: Miscellaneous; G.2.m [Discrete Mathematics]: Miscellaneous

\section{General Terms}

Theory, Algorithms

\section{Keywords}

probability distributions, randomized algorithms, property testing, learning

\section{INTRODUCTION}

Data consisting of samples from distributions is notorious for reliability issues: Sample data can be greatly affected by noise, calibration problems or other faults in the sample recording process; portions of data may be lost; extraneous samples may be erroneously recorded. Such noise may be completely random, or may have some underlying structure. To give a sense of the range of difficulties one might have with sample data, we mention some examples: A sensor network which tracks traffic data may have dead sensors which transmit no data at all, or other sensors that are defective and transmit arbitrary numbers. Sample data from surveys may suffer from response rates that are correlated with location or socioeconomic factors. Sample data from species distribution models are prone to geographic location errors [28].

Statisticians have grappled with defining a methodology for working with distributions in the presence of noise by correcting the samples. If, for example, you know that the uncorrupted distribution is Gaussian, then it would be natural to correct the samples of the distribution to the nearest Gaussian. The challenge in defining this methodology is: how do you correct the samples if you do not know much about the original uncorrupted distribution? To analyze 
distributions with noise in a principled way, approaches have included imputation [34, 41, 38] for the case of missing or incomplete data, and outlier detection and removal $[27,3$, $29]$ to handle "extreme points" deviating significantly from the underlying distribution. More generally, the question of coping with the sampling bias inherent to many strategies (such as opportunity sampling) used in studying rare events or species, or with inaccuracies in the reported data, is a key challenge in many of the natural and social sciences (see e.g. $[43,42,36])$. While these problems are usually dealt with drawing on additional knowledge or by using specific modeling assumptions, no general procedure is known that addresses them in a systematic fashion.

In this work, we propose a methodology which is based on using known structural properties of the distribution to design sampling correctors which "correct" the sample data. While assuming these structural properties is in itself a type of modeling, it is in general much weaker than postulating a strict form of the data (e.g., that it follows a linear model perturbed by Gaussian noise). Examples of structural properties which might be used to correct samples include the property of being bimodal, a mixture of several Gaussians, or an independent joint distribution. Within this methodology, the main question is: how best can one output samples of a distribution in which on one hand, the structural properties are restored, and on the other hand, the corrected distribution is close to the original distribution? We show that this task is intimately connected to distribution learning tasks, but we also give instances in which such tasks can be performed strictly more efficiently.

\subsection{Our model}

We introduce two (related) notions of algorithms to correct distributions: sampling correctors and sampling improvers. Although the precise definitions are deferred to Section 2, we describe and state informally what we mean by these. In what follows, $\mathcal{X}$ is a finite domain, $\mathcal{P}$ is any fixed property of distributions, i.e., a subset of distributions, over $\mathcal{X}$ and distances between distributions are measured according to their total variation distance. ${ }^{1}$

A sampling corrector for $\mathcal{P}$ is a randomized algorithm which gets samples from a distribution $D$ guaranteed to be $\varepsilon$-close to having property $\mathcal{P}$, and outputs a sample from a "corrected distribution" $\tilde{D}$ which, with high probability, (a) has the property; and (b) is still close to the original distribution $D$ (i.e., within distance $\varepsilon_{1}$ ). The sample complexity of such a corrector is the number of samples it needs to obtain from $D$ in order to output one from $\tilde{D}$.

To make things concrete, we give a simple example of correcting independence of distributions over a product space $[n] \times[m]$. For each pair of samples $(x, y)$ and $\left(x^{\prime}, y^{\prime}\right)$ from a distribution $D$ which is $\varepsilon$-close to independent, output one sample $\left(x, y^{\prime}\right)$. As $x$ and $y^{\prime}$ are independent, the resulting distribution clearly has the property; and it can be shown that if $D$ was indeed $\varepsilon$-close to independent, then the distribution of $\left(x, y^{\prime}\right)$ will indeed be $3 \varepsilon$-close to $D$ [39]. (Whether this sample complexity can be reduced further to $q<2$, even on average, is an open question.)

Note that in some settings it may be too much to ask for complete correction (or may even not be the most desirable

\footnotetext{
${ }^{1}$ The total variation distance is defined as $\mathrm{d}_{\mathrm{TV}}\left(D_{1}, D_{2}\right) \stackrel{\text { def }}{=}$ $\max _{S \subseteq \mathcal{X}}\left(D_{1}(S)-D_{2}(S)\right)=\frac{1}{2} \sum_{x \in \mathcal{X}}\left|D_{1}(x)-D_{2}(x)\right|$.
}

option). For this reason, we also consider the weaker notion of sampling improvers, which is similar to a sampling corrector but is only required to transform the distribution into a new distribution which is closer to having the property $\mathcal{P}$.

One naive way to solve these problems, the "learning approach," is to approximate the probability mass function of $D$, and find a candidate $\tilde{D} \in \mathcal{P}$. Since we assume we have a complete description of $\tilde{D}$, we can then output samples according to $\tilde{D}$ without further access to $D$. In general, such an approach can be very inefficient in terms of time complexity. However, if there is an efficient agnostic proper learning algorithm ${ }^{2}$ for $\mathcal{P}$, we show that this approach can lead to efficient sampling correctors. For example, we use such an approach to give sampling correctors for the class of monotone distributions.

In our model, we focus on optimizing the following two parameters of our correcting algorithms: The first parameter is the number of samples of $D$ needed to output samples of $\tilde{D}$. The second parameter is the number of additional truly random bits needed for outputting samples of $\tilde{D}$. Note that in the above learning approach, the dependence on each of these parameters could be quite large. Although these parameters are not independent of each other (if $D$ is of high enough entropy, then it can be used to simulate truly random bits), they can be thought of as complementary, as one typically will aim at a tradeoff between the two. Furthermore, a parsimonious use of extra random bits may be crucial for some applications, while in others the correction of the data itself is the key factor; for this reason, we track each of the parameters separately. For any property $\mathcal{P}$, the main question is whether one can achieve improved complexity in terms of these parameters over the use of the naive (agnostic) learning approach for $\mathcal{P}$.

\subsection{Our results}

In this work, we focus on two particular properties of interest, namely uniformity and monotonicity. The first one, arguably one of the most natural and illustrative properties to be considered, is nonetheless deeply challenging in the setting of randomness scarcity. As for the second, not only does it provide insight in the workings of sampling correctors as well as non-trivial connections and algorithmic results, but is also one of the most-studied classes of distributions in the statistics and probability literature, with a body of work covering several decades (see e.g. [26, 9, 7, 21], or [22] for a detailed list of references). Moreover, recent work on distribution testing $[22,13]$ shows strong connections between monotonicity and a wide range of other properties, such as for instance log-concavity, Monotone Hazard Risk and Poisson Binomial Distributions. This gives evidence that the study of monotone distributions may have direct implications for correction of many of these "shape-constrained properties."

\footnotetext{
${ }^{2}$ Recall that a learning algorithm for a class of distributions $\mathcal{C}$ is an algorithm which gets independent samples from an unknown distribution $D \in \mathcal{C}$; and on input $\varepsilon$ must, with high probability, output a hypothesis which is $\varepsilon$-close to $D$ in total variation distance. If the hypotheses the algorithm produces are guaranteed to belong to $\mathcal{C}$ as well, we call it a proper learning algorithm. Finally, if the - not-necessarily proper - algorithm is able to learn distributions that are only close to $\mathcal{C}$, returning a hypothesis at a distance at most OPT $+\varepsilon$ from $D-$ where OPT is the distance from $D$ to the class, it is said to be agnostic. For a formal definition of these concepts, the reader is referred to the full version.
} 


\section{Sampling correctors, learning algorithms and property testing algorithms.}

We begin by showing implications of the existence of sampling correctors for the existence of various types of learning and property testing algorithms in other models. We first show in Theorem 3.1 that learning algorithms for a distribution class imply sampling correctors for distributions in this class (under any property to correct) with the same sample complexity, though not necessarily the same running time dependency. However, when efficient agnostic proper learning algorithms for a distribution class exist, we show that there are efficient sampling correctors for the same class. In [9, 18] efficient algorithms for agnostic learning of concise representations for several families of distributions are given, including distributions that are monotone, $k$-histograms, Poisson binomial, and sums of $k$ independent random variables. Not all of these algorithms are proper.

Next, we show in Theorem 3.3 that the existence of (a) an efficient learning algorithm, as e.g. in [31, 17, 20, 19], and (b) an efficient sampling corrector for a class of distributions implies an efficient agnostic learning algorithm for the same class of distributions. It is well known that agnostic learning can be much harder than non-agnostic learning, as in the latter the algorithm is able to leverage structural properties of the class $\mathcal{C}$. Thus, by the above result we also get that any agnostic learning lower bounds can be used to obtain sampling corrector lower bounds.

Our third result in this section, Theorem 3.5, shows that an efficient property tester, an efficient distance estimator (which computes an additive estimate of the distance between two distributions) and an efficient sampling corrector for a distribution class imply a tolerant property tester with complexity equal to the complexity of correcting the number of samples required to run both the tester and estimator. ${ }^{3}$ As tolerant property testing can be much more difficult than property testing $[25,6,35,46]$, this gives a general purpose way of getting both upper bounds on tolerant property testing and lower bounds on sampling correctors.

We describe how these results can be employed in Section 3, where we give specific applications in achieving improved property testers for various properties.

\section{Is sampling correction easier than learning?.}

We next turn to the question of whether there are natural examples of sampling correctors whose query complexity is much smaller than that of distribution learning algorithms for the same class. While the sample complexity of learning monotone distributions is known to be $\Omega(\log n)$ [9] (this lower bound on the sample and query complexity holds even when the algorithm is allowed both to make queries to the cumulative distribution function as well as to access samples of the distribution), we present an oblivious sampling corrector for monotone distributions with sample complexity $O(1)$, which corrects error smaller than $\varepsilon \leq O\left(1 / \log ^{2} n\right)$. This is done by first implicitly approximating the distribution by a "histogram" on only a small number of intervals, using ingredients from [9]. This (very close) approximation can then be combined, still in an oblivious way, with a carefully

\footnotetext{
${ }^{3}$ Recall that the difference between testing and tolerant testing lies in that the former asks to distinguish whether an unknown distribution has a property, or is far from it, while the latter requires to decide whether the distribution is close to the property versus far from it.
}

chosen slowly decreasing distribution, so that the resulting mixture is not only guaranteed to be monotone, but also close to the original distribution.

It is open whether there exist sampling correctors for monotone distributions with sample complexity $o\left((\log n) / \varepsilon^{3}\right)$ that can correct arbitrary error $\varepsilon \in(0,1)$, thus beating the sample complexity of the "learning approach." (We note however that a logarithmic dependence on $n$ is inherent when $\varepsilon=\omega(1 / \log n)$, as pointed out to us by Paul Valiant [47].)

Assuming a stronger type of access to the unknown distribution - namely, query access to its cumulative distribution function $(\mathrm{CDF})$ as in $[4,15]$, we describe in Section 4 a sampling corrector for monotonicity with (expected) query complexity $O(\sqrt{\log n})$ which works for arbitrary $\varepsilon \in(0,1)$. At a high-level, our algorithm combines the "succinct histogram" technique mentioned above with a two-level bucketing approach to correct the distribution first at a very coarse level only (on "superbuckets"), and defer the finer corrections (within a given superbucket) to be made on-the-go at query time. The challenge in this last part is that one must ensure that all of these disjoint local corrections are consistent with each other - and crucially, with all future sample corrections. To achieve this, we use a "boundary correction" subroutine which fixes potential violations between two neighboring superbuckets by evening out the boundary differences. To make it possible, we use rejection sampling to allocate adaptively an extra "budget" to each superbucket that this subroutine can use for corrections.

\section{Restricted error models.}

Since many of the sampling correction problems are difficult to solve in general, we suggest error models for which more efficient sampling correction algorithms may exist. A first class of error models, which we refer to as missing data errors, is introduced in the full version of this paper and defined as follows - given a distribution over $[n]$, all samples in some interval $[i, j]$ for $1<i<j<n$ are deleted. Such errors could correspond to samples from a sensor network where one of the sensors ran out of power; emails mistakenly deleted by a spam filter; or samples from a study in which some of the paperwork got lost. Whenever the input distribution $D$, whose distance from monotonicity is $\varepsilon \in(0,1)$, falls under this model, we give a sampling improver that is able to find a distribution both $\varepsilon_{2}$-close to monotone and $O(\varepsilon)$-close to the original using $\tilde{O}\left(1 / \varepsilon_{2}^{3}\right)$ samples. The improver works in two stages. In the "preprocessing stage," we detect the location of the missing interval (when the missing weight is sufficiently large) and then estimate its missing weight, using a "learning through testing" approach from [21] to keep the sample complexity under control. In the second stage, we give a procedure by which the algorithm can use its knowledge of the estimated missing interval to correct the distribution by rejection sampling.

\section{Randomness Scarcity.}

We then consider the case where only a limited amount of randomness (other than the input distribution) is available, and optimizing its use, possibly at the cost of worse parameters and/or sample complexity of our sampling improvers, is crucial. This captures situations where generating 
the random bits the algorithm use is either expensive ${ }^{4}$ (as in the case of physical implementations relying on devices, such as Geiger counters or Zener diodes) or undesirable (e.g., when we want the output distribution to be a deterministic function of the input data, for the sake of reproducibility or parallelization). We focus on this setting in the second part of the full version, and provide sampling correctors and improvers for uniformity that use samples only from the input distribution. For example, we give a sampling improver that, given access to distribution $\varepsilon$-close to uniform, grants access to a distribution $\varepsilon_{2}$-close to uniform distribution and has constant sample complexity $O_{\varepsilon, \varepsilon_{2}}(1)$. We achieve this by exploiting the fact that the uniform distribution is not only an absorbing element for convolution in Abelian groups, but also an attractive fixed point with high convergence rate. That is, by convolving a distribution with itself (i.e., summing independent samples modulo the order of the group) one gets very quickly close to uniform. Combining this idea with a different type of improvement (based on a von Neumann-type "trick") allows us to obtain an essentially optimal tradeoff between closeness to uniform and to the original distribution.

\subsection{Open problems}

Correcting vs. Learning. A main direction of interest would be to obtain more examples of properties for which correcting is strictly more efficient than (agnostic or nonagnostic) learning. Such examples would be insightful even if they are more efficient only in terms of the number of samples required from the original distribution, without considering the additional randomness requirements for generating the distribution. More specifically, one may ask whether there exists a sampling corrector for monotonicity of distributions (i.e., one that beats the learning bound from Theorem 4.1) for all $\epsilon<1$ which uses at most $o\left((\log n) / \varepsilon^{3}\right)$ samples from the original distribution per sample output of the corrected distribution. Other properties of interest, among many, include log-concavity of distributions, having a piecewise-constant density (i.e., being a $k$-histogram for some fixed value $k$ ), or being a Poisson Binomial Distribution.

The power of additional queries. Following the line of work pursued in $[16,14,15]$ (in the setting of distribution testing), it is natural in many situations to consider additional types of queries to the input distribution: e.g., either conditional queries (getting a sample conditioned on a specific subset of the domain) or cumulative queries (granting query access to the cumulative distribution function, besides the usual sampling). By providing algorithms with this extended access to the underlying probability distribution, can one obtain faster sampling correctors for specific properties, as we do in the case of monotonicity?

Confidence boosting. Suppose that there exists, for some property $\mathcal{P}$, a sampling improver $\mathcal{A}$ that only guarantees a success probability ${ }^{5}$ of $2 / 3$. Using $\mathcal{A}$ as a black-box, can

\footnotetext{
${ }^{4}$ On this topic, see for instance the discussion in $[33,30]$, and references therein.

${ }^{5}$ We note that the case of interest here is of batch sampling improvers: indeed, in order to generate a single draw, a sampling improver acts in a non-trivial way only if the parameter $\varepsilon$ is greater than its failure probability $\delta$. If not, a draw from the original distribution already satisfies the requirements.
}

one design a sampling improver $\mathcal{A}^{\prime}$ which succeeds with probability $1-\delta$, for any $\delta$ ?

More precisely, let $\mathcal{A}$ be a batch improver for $\mathcal{P}$ which, when queried, makes $q\left(\varepsilon_{1}, \varepsilon_{2}\right)$ queries and provides $t \geq 1$ samples, with success probability at least $2 / 3$. Having blackbox access to $\mathcal{A}$, can we obtain a batch improver $\mathcal{A}^{\prime}$ which on input $\delta>0$ provides $t^{\prime} \geq 1$ samples, with success probability at least $1-\delta$ ? If so, what is the best $t^{\prime}$ one can achieve, and what is the minimum query complexity of $\mathcal{A}^{\prime}$ one can get (as a function of $q(\cdot, \cdot), t^{\prime}$ and $\left.\delta\right)$ ?

This is known for property testing (by running the testing algorithm independently $O(\log (1 / \delta))$ times and taking the majority vote), as well as for learning (again, by running the learning algorithm many times, and then doing hypothesis testing, e.g. à la [23] (Theorem 19)). However, these approaches do not straightforwardly generalize to sampling improvers or correctors, respectively because the output is not a single bit, and as we only obtain a sequence of samples (instead of an actual, fully-specified hypothesis distribution).

\subsection{Other previous work}

Dealing with noisy or incomplete datasets has been a challenge in Statistics and data sciences, and many methods have been proposed to handle them. Such methods include imputation and multiple imputation [37], which creates new datasets from incomplete datasets by filling in the missing values randomly according to a maximum likelihood distribution computed from the observations and a modeling assumption made on the data. The parameters of this model are then updated in a manner that resembles the Expectation-Maximization (EM) algorithm [24, 41, 34].

From a Theoretical Computer Science perspective, the problem of local correction of data has received much attention (some examples include [10, 48, 2, 40, 8, 32]). To the best of our knowledge, this is the first work to address the correction of data from distributions.

It is instructive to compare the goal of our model of distribution sampling correctors to that of extractors: in spite of many similarities, the two have essential differences and the results are in many cases incomparable. We defer this discussion to the full version.

\section{Organization.}

After stating the formal definition of our model in Section 2, we draw in Section 3 some connections it yields to learning and testing. Section 4 focuses on the sample complexity of correcting monotonicity, and contains three different algorithmic results for this problem. as well as a corrector for the "missing data error" model. Due to space constraints, our results on randomness scarcity and all proofs are deferred to the full version.

\section{OUR MODEL: DEFINITIONS}

In this section, we state the precise definitions of sampling correctors, improvers and batch sampling improvers. To get an intuition, the reader may think for instance of the parameter $\varepsilon_{1}$ below as being $2 \varepsilon$, and the error probability $\delta$ as $1 / 3$. Although all definitions are presented in terms of the total variation distance, analogous definitions in terms of other distances can also be made.

Definition 2.1 (SAmpling Corrector). Fix a given property $\mathcal{P}$ of distributions on $\mathcal{X}$. An $\left(\varepsilon, \varepsilon_{1}\right)$-sampling correc- 
tor for $\mathcal{P}$ is a randomized algorithm which is given parameters $\varepsilon, \varepsilon_{1} \in(0,1]$ such that $\varepsilon_{1} \geq \varepsilon$ and $\delta \in[0,1]$, as well as sampling access to a distribution $D$. Under the promise that $\mathrm{d}_{\mathrm{TV}}(D, \mathcal{P}) \leq \varepsilon$, the algorithm must provide, with probability at least $1-\delta$ over the samples it draws and its internal randomness, sampling access to a distribution $\tilde{D}$ such that

(i) $\tilde{D}$ is close to $D: \mathrm{d}_{\mathrm{TV}}(\tilde{D}, D) \leq \varepsilon_{1}$;

(ii) $\tilde{D}$ has the property: $\tilde{D} \in \mathcal{P}$.

In other terms, with high probability the algorithm will simulate exactly a sampling oracle for $\tilde{D}$. The query complexity $q=q\left(\varepsilon, \varepsilon_{1}, \delta, \mathcal{X}\right)$ of the algorithm is the number of samples from $D$ it takes per query in the worst case.

One can define a more general notion, which allows the algorithm to only get "closer" to the desired property, and convert some type of access ORACLE ${ }_{1}$ into some other type of access $\mathrm{ORACLE}_{2}$ (e.g., from sampling to evaluation access):

DeFinition 2.2 (SAmpling IMPROVER). Fix a given property $\mathcal{P}$ over distributions on $\mathcal{X}$. A sampling improver for $\mathcal{P}$ (from ORACLE $\mathrm{O}_{1}$ to $\mathrm{ORACLE}_{2}$ ) is a randomized algorithm which, given parameter $\varepsilon \in(0,1]$ and $\mathrm{ORACLE}_{1}$ access to a distribution $D$ with the promise that $\mathrm{d}_{\mathrm{TV}}(D, \mathcal{P}) \leq \varepsilon$ as well as parameters $\varepsilon_{1}, \varepsilon_{2} \in[0,1]$ satisfying $\varepsilon_{1}+\varepsilon_{2} \geq \varepsilon$, provides, with probability at least $1-\delta$ over the answers from $\mathrm{ORACLE}_{1}$ and its internal randomness, ORACLE ${ }_{2}$ access to a distribution $\tilde{D}$ such that

$$
\begin{aligned}
& \mathrm{d}_{\mathrm{TV}}(\tilde{D}, D) \leq \varepsilon_{1} \\
& \mathrm{~d}_{\mathrm{TV}}(\tilde{D}, \mathcal{P}) \leq \varepsilon_{2}
\end{aligned}
$$

(Close to $D)$

$($ Close to $\mathcal{P})$

In other terms, with high probability the algorithm will simulate exactly $\mathrm{ORACLE}_{2}$ access to $\tilde{D}$. The query complexity $q=q\left(\varepsilon, \varepsilon_{1}, \varepsilon_{2}, \delta, \mathcal{X}\right)$ of the algorithm is the number of queries it makes to ORACLE ${ }_{1}$ in the worst case.

Finally, one may ask for such an improver to provide many samples from the (same) improved distribution, ${ }^{6}$ where "many" is a number committed in advance. We refer to such an algorithm as a batch sampling improver (or, similarly, batch sampling corrector):

Definition 2.3 (Batch Sampling Improver). For $\mathcal{P}$, $D, \varepsilon, \varepsilon_{1}, \varepsilon_{2} \in[0,1]$ as above, and parameter $m \in \mathbb{N}$, a batch sampling improver for $\mathcal{P}$ (from ORACLE Oro $_{1}$ ORACLE ${ }_{2}$ ) is a sampling improver which provides, with probability at least $1-\delta$, $\mathrm{ORACLE}_{2}$ access to $\tilde{D}$ for as many as $m$ queries, in between which it is allowed to maintain some internal state ensuring consistency. The query complexity of the algorithm is now allowed to depend on $m$ as well.

Note that, in particular, when providing sampling access to $\tilde{D}$ the batch improver must guarantee independence of the $m$ samples. When $\varepsilon_{2}$ is set to 0 in the above definition, we will refer to the algorithm as a batch sampling corrector.

REMARK 2.4 (ON PARAMETERS OF INTEREST). We observe that the regime of interest of our correctors and improvers is when the number of corrected samples to output is

\footnotetext{
${ }^{6}$ Indeed, observe that as sampling correctors and improvers are randomized algorithms with access to their "own" coins, there is no guarantee that fixing the input distribution $D$ would lead to the same output distribution $\tilde{D}$.
}

at least of the order $\Omega(1 / \varepsilon)$. Indeed, if fewer samples are required, then the assumption that the distribution $D$ be $\varepsilon$-close to having the property implies that - with high probability a small number of samples from $D$ will be indistinguishable from the closest distribution having the property. (So that, intuitively, they are already "as good as it gets," and need not be corrected.)

REMARK 2.5 (ON TESTING LOWER BOUNDS). A similar observation holds for properties $\mathcal{P}$ that are known to be hard to test, that is for which some lower bound of $q(n, \varepsilon)$ samples holds to decide whether a given distribution satisfies $\mathcal{P}$, or is $\varepsilon$-far from it. In light of such a lower bound, one may wonder whether there is something to be gained in correcting $m<q(n, \varepsilon)$ samples, instead of simply using $m$ samples from the original distribution altogether. However, such a result only states that there exists some worst-case instance $D^{*}$ that $i s$ at distance $\varepsilon$ from the property $\mathcal{P}$, yet requires this many samples to be distinguished from it: so that any algorithm relying on samples from distributions satisfying $\mathcal{P}$ could be fed $q(n, \varepsilon)-1$ samples from this particular $D^{*}$ without complaining. Yet, for "typical" distributions that are $\varepsilon$-close to $\mathcal{P}$, it may be that far fewer samples are required to reveal their deviation from it: for many, as few as $O(1 / \varepsilon)$ suffice. Thus, an algorithm that expects to get say $q(n, \varepsilon)^{99}$ samples from a honest-to-goodness distribution from $\mathcal{P}$, but instead is provided with samples from one that is merely $\varepsilon$-close to it, may break down very quickly. Our corrector, in this very regime of $o(q(n, \varepsilon))$ samples, guarantees this will not happen.

\section{CONNECTIONS TO LEARNING AND TEST- ING}

In this section, we draw connections between sampling improvers and other areas, namely testing and learning. These connections shed light on the relation between our model and these other lines of work, and provide a way to derive new algorithms and impossibility results for both testing or learning problems.

\subsection{From learning to correcting}

As a first observation, it is not difficult to see that, under the assumption that the unknown distribution $D$ belongs to some specific class $\mathcal{C}$, correcting (or improving) a property $\mathcal{P}$ requires at most as many samples as learning the class $\mathcal{C}$; that is, learning (a class of distributions) is at least as hard as correcting (distributions of this class). Here, $\mathcal{P}$ and $\mathcal{C}$ need not be related.

Indeed, assuming there exists a learning algorithm $\mathcal{L}$ for $\mathcal{C}$, it suffices to run it on the unknown distribution $D \in \mathcal{C}$ to learn a hypothesis $\hat{D}$ sufficiently close to $D$. In particular, $\hat{D}$ must itself still be close to $\mathcal{P}$. One can then (e.g., by exhaustive search) find $\tilde{D}$ in $\mathcal{P}$ which is closest to $\hat{D}$ (and therefore at most $\varepsilon_{1}$-far from $D$ ), and use it to produce as many "corrected samples" as wanted:

THEOREM 3.1. Let $\mathcal{C}$ a class of probability distributions over $\mathcal{X}$. Suppose there exists a learning algorithm $\mathcal{L}$ for $\mathcal{C}$ with sample complexity $q_{\mathcal{L}}$. Then, for any property $\mathcal{P}$ of distributions, there exists a (not-necessarily computationally efficient) sampling corrector for $\mathcal{P}$ with sample complexity $q\left(\varepsilon, \varepsilon_{1}, \delta\right)=q_{\mathcal{L}}\left(\frac{\varepsilon_{1}-\varepsilon}{2}, \delta\right)$, under the promise that $D \in \mathcal{C}$.

Furthermore, if the (efficient) learning algorithm $\mathcal{L}$ has the additional guarantee that the hypotheses it outputs always 
belong to (a subset of) $\mathcal{P}$, and allow efficient generation of samples, then we immediately obtain a computationally efficient sampling corrector: indeed, in this case $\hat{D} \in \mathcal{P}$ already. Furthermore, as mentioned in the introduction, when efficient agnostic proper learning algorithms for distribution classes exist, then there are efficient sampling correctors for the same classes. Yet, it is worth pointing out that this correcting-by-learning approach is quite inefficient with regard to the amount of extra randomness needed: indeed, every sample generated from $\tilde{D}$ requires fresh new random bits.

To illustrate our theorem, we give here an easy corollary. This result follows from Chan et al., who showed in [17] that monotone hazard risk distributions can be learned to accuracy $\varepsilon$ using $\tilde{O}\left(\log n / \varepsilon^{4}\right)$ samples; moreover, the hypothesis obtained is a $O\left(\log (n / \varepsilon) / \varepsilon^{2}\right)$-histogram.

Corollary 3.2. Let $\mathcal{C}$ be the class of monotone hazard risk distributions over $[n]$, and $\mathcal{P}$ be the property of being a histogram with (at most) $\sqrt{n}$ pieces. Then, under the promise that $D \in \mathcal{C}$ and as long as $\varepsilon=\tilde{\Omega}(1 / \sqrt{n})$, there is a sampling corrector for $\mathcal{P}$ with sample complexity $\tilde{O}\left(\frac{\log n}{\left(\varepsilon_{1}-\varepsilon\right)^{4}}\right)$.

\subsection{From correcting to agnostic learning}

Let $\mathcal{C}$ and $\mathcal{H}$ be two classes of probability distributions over $\mathcal{X}$. Recall that a (semi-)agnostic learner for $\mathcal{C}$ (using hypothesis class $\mathcal{H}$ ) is a learning algorithm $\mathcal{A}$ which, given sample access to an arbitrary distribution $D$ and parameter $\varepsilon$, outputs a hypothesis $\hat{D} \in \mathcal{H}$ such that, with high probability, $\hat{D}$ does "as much as well as the best approximation from $\mathcal{C}: " \mathrm{~d}_{\mathrm{TV}}(D, \hat{D}) \leq c \cdot \operatorname{OPT}_{\mathcal{C}, D}+O(\varepsilon)$, where $\operatorname{OPT}_{\mathcal{C}, D}=\inf _{D_{\mathcal{C}} \in \mathcal{C}} \mathrm{d}_{\mathrm{TV}}\left(D_{\mathcal{C}}, D\right)$ and $c \geq 1$ is some absolute constant (if $c=1$, the learner is said to be agnostic).

In the full version, we first describe how to combine a (non-agnostic) learning algorithm with a sampling corrector in order to obtain an agnostic learner, under the strong assumption that a (rough) estimate of OPT is known. Then, we explain how to get rid of this extra requirement, using machinery from the distribution learning literature (namely, an efficient hypothesis selection procedure). This leads to the following result:

TheOREM 3.3. Let $\mathcal{C}$ be as above. Suppose there exists a learning algorithm $\mathcal{L}$ for $\mathcal{C}$ with sample complexity $q_{\mathcal{L}}$, and a batch sampling corrector $\mathcal{A}$ for $\mathcal{C}$ with sample complexity $q_{\mathcal{A}}$. Suppose further that a constant-factor estimate $\widehat{\mathrm{OPT}}$ of $\mathrm{OPT}_{\mathcal{C}, D}$ is known (up to a multiplicative $c$ ).

Then, there exists a (semi) agnostic learner for $\mathcal{C}$ with sample complexity $q(\varepsilon, \delta)=q_{\mathcal{A}}\left(\widehat{\mathrm{OPT}}, \widehat{\mathrm{OPT}}+\varepsilon, q_{\mathcal{L}}\left(\varepsilon, \frac{\delta}{2}\right), \frac{\delta}{2}\right)$ (where the constant in front of $\mathrm{OPT}_{\mathcal{C}, D}$ is c). Moreover, if such $\widehat{\mathrm{OPT}}$ is not known, the result still holds at the price of a polylog $(1 / \varepsilon)$ blowup in the sample complexity.

It is worth noting that in the case the learning algorithm is proper (meaning the hypotheses it outputs belong to the target class $\mathcal{C}$ : that is, $\mathcal{H}=\mathcal{C}$ ), then so is the agnostic learner obtained with Theorem 3.3. This turns out to be a very strong guarantee: specifically, getting (computationally efficient) proper agnostic learning algorithms remains a challenge for many classes of interest - see e.g. [20], which mentions efficient proper learning of Poisson Binomial Distributions as an open problem.
We stress that the above can be viewed as a generic framework to obtain efficient agnostic learning results from known efficient learning algorithms. For the sake of illustration, let us consider the simple case of Binomial distributions: it is known, for instance as a consequence of the aforementioned results on $\mathrm{PBDs}$, that learning such distributions can be performed with $\tilde{O}\left(1 / \varepsilon^{2}\right)$ samples (and that $\Omega\left(1 / \varepsilon^{2}\right)$ are required). Our theorem then provides a simple way to obtain agnostic learning of Binomial distributions with sample complexity $\tilde{O}\left(1 / \varepsilon^{2}\right)$ : namely, by designing an efficient sampling corrector for this class with sample complexity poly $\left(\log \frac{1}{\varepsilon}, \log \frac{1}{\varepsilon_{1}}\right)$. (Since publication of our work, we have learned that [1] provides such a result unconditionally.)

COROLlary 3.4. Suppose there exists a batch sampling corrector $\mathcal{A}$ for the class $\mathcal{B}$ of binomial distributions over $[n]$, with sample complexity $q_{\mathcal{A}}\left(\varepsilon, \varepsilon_{1}, m, \delta\right)=\operatorname{polylog}\left(\frac{1}{\varepsilon}, \frac{1}{\varepsilon_{1}}, m, \frac{1}{\delta}\right)$. Then, there exists a semi-agnostic learner for $\mathcal{B}$, which, given access to an unknown distribution $D$ promised to be $\varepsilon$-close to some binomial distribution, takes $\tilde{O}\left(\frac{1}{\varepsilon^{2}}\right)$ samples from $D$ and outputs a distribution $\hat{B} \in \mathcal{B}$ such that

$$
\mathrm{d}_{\mathrm{TV}}(D, \hat{B}) \leq 3 \varepsilon
$$

with probability at least $2 / 3$.

To the best of our knowledge, an agnostic learning algorithm for the class of Binomial distributions with sample complexity $\tilde{O}\left(1 / \varepsilon^{2}\right)$ is not explicitly known, although the results of [18] do imply a $\tilde{O}\left(1 / \varepsilon^{3}\right)$ upper bound and a modification of [20] (to make their algorithm agnostic) seems to yield one. The above suggests an approach which would lead to the (essentially optimal) sample complexity.

\subsection{From correcting to tolerant testing}

We observe that the existence of sampling correctors for a given property $\mathcal{P}$, along with an efficient distance estimation procedure, allows one to convert any distribution testing algorithm into a tolerant distribution testing algorithm. This is similar to the connection between "local reconstructors" and tolerant testing of graphs described in [11] (Theorem 3.1 ) and [12] (Theorem 3.1). That is, if a property $\mathcal{P}$ has both a distance estimator and a sampling corrector, then one can perform tolerant testing of $\mathcal{P}$ in the time required to generate enough corrected samples for both the estimator and a (non-tolerant) tester.

We first state our theorem in all generality, before instantiating it in several corollaries. For the sake of clarity, the reader may wish to focus on these on a first pass.

THEOREM 3.5. Let $\mathcal{C}$ be a class of distributions, and $\mathcal{P} \subseteq$ $\mathcal{C}$ a property. Suppose there exists an $\left(\varepsilon, \varepsilon_{1}\right)$-batch sampling corrector $\mathcal{A}$ for $\mathcal{P}$ with complexity $q_{\mathcal{A}}$, and a distance estimator $\mathcal{E}$ for $\mathcal{C}$ with complexity $q_{\mathcal{E}}$ - that is, given sample access to $D_{1}, D_{2} \in \mathcal{C}$ and parameters $\varepsilon, \delta, \mathcal{E}$ draws $q_{\mathcal{E}}(\varepsilon, \delta)$ samples from $D_{1}, D_{2}$ and outputs a value $\hat{d}$ such that $\left|\hat{d}-\mathrm{d}_{\mathrm{TV}}\left(D_{1}, D_{2}\right)\right| \leq \varepsilon$ with probability at least $1-\delta$.

Then, from any property tester $\mathcal{T}$ for $\mathcal{P}$ with sample complexity $q_{\mathcal{T}}$, one can get a tolerant tester $\mathcal{T}^{\prime}$ with query complexity $q\left(\varepsilon^{\prime}, \varepsilon, \delta\right)=q_{\mathcal{A}}\left(\varepsilon^{\prime}, \Theta(\varepsilon), q_{\mathcal{E}}\left(\frac{\varepsilon-\varepsilon^{\prime}}{4}, \frac{\delta}{3}\right)+q_{\mathcal{T}}\left(\frac{\varepsilon-\varepsilon^{\prime}}{4}, \frac{\delta}{3}\right), \frac{\delta}{3}\right)$.

REMARK 3.6. Only asking that the distance estimation procedure $\mathcal{E}$ be specific to the class $\mathcal{C}$ is not innocent; indeed, 
it is known ([46]) that for general distributions, distance estimation has sample complexity $\Omega\left(n^{1-o(1)}\right)$. However, the task becomes significantly easier for certain classes of distributions: and for instance can be performed with only $\tilde{O}(k \log n)$ samples, if the distributions are guaranteed to be $k$-modal [22]. This observation can be leveraged in cases when one knows that the distribution has a specific property, but does not quite satisfy a second property: e.g. is known to be k-modal but not known to be, say, log-concave.

The reduction above can be useful both as a black-box way to derive upper bounds for tolerant testing, as well as to prove lower bounds for either testing or distance estimation. For the first use, we give two applications of our theorem to provide tolerant monotonicity testers for $k$-modal distributions. The first is a conditional result, showing that the existence of good monotonicity correctors yield tolerant testers. The second, while unconditional, only guarantees a weaker form of tolerance (guaranteeing acceptance only of distributions that are very close to monotone); and relies on a corrector we describe in Section 4. As we detail shortly after stating these two results, even this weak tolerance improves upon the one provided by currently known testing algorithms.

Corollary 3.7. Suppose there exists an $\left(\varepsilon, \varepsilon_{1}\right)$-batch sampling corrector for monotonicity with complexity $q$. Then, for any $k=O(\log n / \log \log n)$, there exists an algorithm that distinguishes whether a $k$-modal distribution is (a) $\varepsilon$-close to monotone or (b) $5 \varepsilon$-far from monotone with success probability $2 / 3$, and sample complexity $q\left(\varepsilon, 2 \varepsilon, C \frac{k \log n}{\varepsilon^{4} \log \log n}, \frac{1}{9}\right)$ where $C$ is an absolute constant.

Another application of this theorem, but this time taking advantage of a result from Section 4, allows us to derive an explicit tolerant tester for monotonicity of $k$-modal distributions:

COROLlary 3.8. For any $k \geq 1$, there exists an algorithm that distinguishes whether a $k$-modal distribution is (a) $O\left(\varepsilon^{3} / \log ^{2} n\right)$-close to monotone or (b) $\varepsilon$-far from monotone with success probability $2 / 3$, and sample complexity $O\left(\frac{1}{\varepsilon^{4}} \frac{k \log n}{\log (k \log n)}+\frac{k^{2}}{\varepsilon^{4}}\right)$. In particular, for $k=O\left(\frac{\log n}{\log \log n}\right)$ this gives a weakly tolerant tester with sample complexity $O\left(\frac{k \log n}{\varepsilon^{4} \log \log n}\right)$.

Note that, to the best of our knowledge, no tolerant tester for monotonicity of $k$-modal distributions was previously known, though using the (regular) $O\left(k / \varepsilon^{2}\right)$-sample tester of [21] and standard arguments, one can achieve a weak tolerance on the order of $O\left(\varepsilon^{2} / k\right)$. While the sample complexity obtained in Corollary 3.8 is worse by a polylog $(n)$ factor, it has better tolerance for $k=\Omega\left(\log ^{2} n / \varepsilon\right)$.

\section{CORRECTING MONOTONICITY}

In this section, we focus on the sample complexity aspect of correcting, considering the specific example of monotonicity correction. As a first result, we show in Theorem 4.1 how to design a simple batch corrector for monotonicity which, after a preprocessing step costing logarithmically many samples, is able to answer an arbitrary number of queries. This corrector follows the "learning approach" described in Section 3.1, and in particular provides a very efficient way to amortize the cost of making many queries to a corrected distribution.
Theorem 4.1 (Correcting By Learning). Fix any constant $c>0$. For any $\varepsilon, \varepsilon_{1} \geq(3+c) \varepsilon$ and $\varepsilon_{2}=0$ as in the definition, any type of oracle ORACLE and any number of queries $m$, there exists a sampling corrector for monotonicity from sampling to ORACLE with sample complexity $O\left(\log n / \varepsilon^{3}\right)$.

A natural question is then whether one can "beat" this approach, and correct the distribution without approximating it as a whole beforehand. Theorem 4.2 answers it by the affirmative: namely, we show that one can correct distributions that are guaranteed to be $\left(1 / \log ^{2} n\right)$-close to monotone in a completely oblivious fashion, with a non-adaptive approach that does not require to learn anything about the distribution.

Theorem 4.2 (OBlivious CORRECTING OF MONOTONICITY). For every $\varepsilon^{\prime} \in(0,1)$, there exists an (oblivious) sampling corrector for monotonicity, with parameters $\varepsilon=O\left(\varepsilon^{\prime 3} / \log ^{2} n\right)$, $\varepsilon_{1}=\varepsilon^{\prime}$ and sample complexity $O(1)$.

Finally, we give with Theorem 4.3 a corrector for monotonicity with no restriction on the range of parameters, but assuming a stronger type of query access to the original distribution. Namely, our algorithm leverages the ability to make $c d f$ queries to the distribution $D$, in order to generate independent samples from a corrected $\tilde{D}$. This sampling corrector also outperforms the one from Theorem 4.1 whenever the number of samples to generate is $o(\log n)$, making only $O(\sqrt{\log n})$ queries per sample on expectation.

THEOREM 4.3. For any $\varepsilon \in(0,1]$, any number of queries $m$ and $\varepsilon_{1}=O(\varepsilon)$ as in the definition, there exists a sampling corrector for monotonicity from Cumulative Dual to SAMP with expected sample complexity $O(\sqrt{m \log n / \varepsilon})$.

\section{High-level idea of the proofs.}

Our first corrector works in a straightforward fashion: it learns a good approximation of the distribution to correct, which is also concisely represented. It then uses this approximation to build a sufficiently good monotone distribution $M^{\prime}$ "offline," by searching for the closest monotone distribution, which in this case can be achieved via linear programming. Any query made to the corrector is then answered according to the latter distribution, at no additional cost.

We next turn to our second monotonicity corrector, which achieves constant sample complexity for distributions already $\left(1 / \log ^{2} n\right)$-close to monotone. ${ }^{7}$ The overall idea is to treat the distribution as a $k$-histogram on the Birgé decomposition (for $k=O(\log n)$ ), thus "implicitly approximating" it; and to correct this histogram by adding a certain amount of probability weight to every interval, so that each gets slightly more than the next one. By choosing these quantities carefully, this ensures that any violation of monotonicity gets corrected

\footnotetext{
${ }^{7}$ Note that while this is a very strong assumption (as samples from such a distribution $D$ are essentially indistinguishable from samples originating from its closest monotone distribution, unless $\Omega\left(\log ^{2} n\right)$ of them are taken already), our construction actually yields a stronger guarantee: namely, given evaluation (query) access to $D$, it can answer evaluation queries to the corrected distribution as well.
} 
in the process, without ever having to find out where they actually occur.

Our next result shows that correcting monotonicity with $o(\log n)$ queries (on expectation) is possible when one allows CDF queries to the original distribution. The main idea of the proof is to first reduce (via the same "Birgé decomposition" technique as before) the problem to that of correcting a histogram supported of logarithmically many intervals, then group these $\ell$ intervals in $K$ "superbuckets," each containing $L=\sqrt{\log n / \varepsilon}$ consecutive intervals from that histogram ("buckets") and finally use a multi-stage approach to correct all monotonicity violations:

Global violation fixing First, we obtain the weights of all superbuckets via $K \mathrm{CDF}$ queries and optimally reweight them so that average weights are non-increasing.

Budget allocation using rejection sampling, we allocate adaptively to each superbucket a "weight budget" it can use to fix on-the-go (at sampling time) boundary violations between adjacent superbuckets.

Local violation fixing we sample a superbucket from the coarse distribution, and use $O(L)$ CDF queries to correct monotonicity of the weights of the $L$ buckets it contains; we also do this for the adjacent superbucket.

Boundary violation fixing to ensure monotonicity at a global scale, we then use the "allocated budget" of the superbucket to fix any possible violation at the boundary with its neighbor.

We stress that the above sweeps many details and difficulties under the rug. For the full description and proofs of the algorithms, the reader is referred to the full version of this extended abstract.

\subsection{A restricted error model: missing data}

In the previous sections, no assumption was made on the form of the error, only on the amount. In this section, we suggest a model of errors capturing the deletion of a whole "chunk" of the distribution. We refer to this model as the missing data model, where we assume that some $\varepsilon$ probability is removed by taking out all the weight of an arbitrary interval $[i, j]$ for $1 \leq i<j \leq n$ and redistributing it on the rest of the domain as per rejection sampling (See Figure 1 for an illustration). ${ }^{8}$ We show that one can design sampling improvers for monotone distributions with arbitrarily large amounts of error.

THEOREM 4.4. For the class of distributions following the "missing data" error model, there exists a batch sampling improver MISSING-DATA-IMPROVER, that, on input $\varepsilon, q, \delta$ and $\alpha$, achieves parameters $\varepsilon_{1}=O(\varepsilon)$ and any $\varepsilon_{2}<\varepsilon$; and has sample complexity $\tilde{O}\left(\frac{1}{\varepsilon_{2}^{3}} \log \frac{1}{\delta}\right)$ independent of $\varepsilon$.

Our sampling improver follows what could be called the "learning-just-enough" approach: instead of attempting to approximate the whole unaltered original distribution, it only tries to learn the values of $i, j$; and then generates samples "on-the-fly." At a high level, the algorithm works

\footnotetext{
${ }^{8}$ That is, if $D$ was the original distribution, the "faulty" one $D^{(i, j)}$ is formally defined as $(1+\varepsilon) \mathbb{1}_{[n] \backslash[i, j]} \cdot D-\varepsilon \cdot \mathcal{U}_{[i, j]}$, where $\varepsilon=D([i, j])$.
}

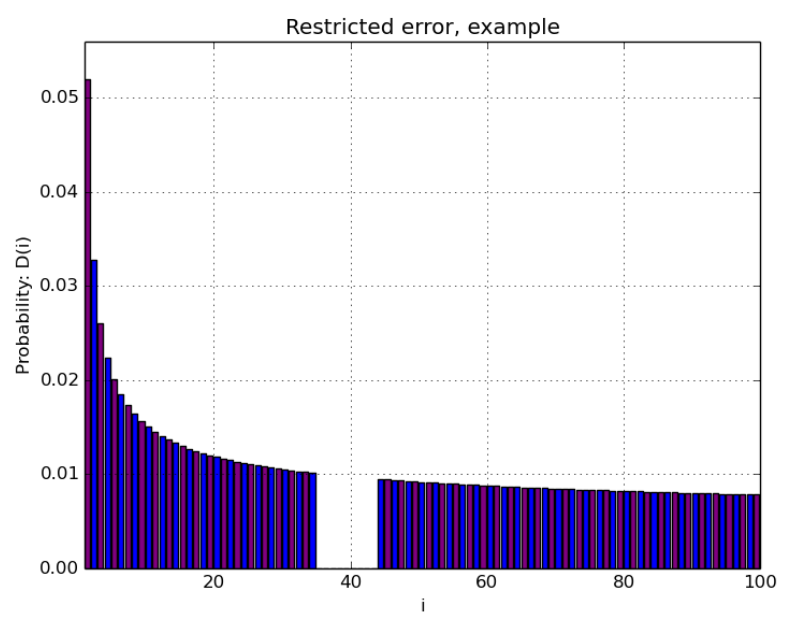

Figure 1: An example of distribution with "missing data" (here, $n=100$ and $[i, j]=[35,43]$ ).

by (i) detecting the location of the missing interval (drawing a large (but still independent of $n$ ) number of samples), then (ii) estimating the weight of this interval under the original, unaltered distribution; and finally (iii) filling this gap uniformly by moving the right amount of probability weight from the end of the domain. To perform the first stage, we follow a paradigm first appeared in [21], and utilize testing as a subroutine to detect "when enough learning has been done." (To efficiently implement this, we can leverage the structure of the error itself, which allows use to circumvent the prohibitive cost of testing monotonicity of general distributions.)

\section{REFERENCES}

[1] J. Acharya, I. Diakonikolas, J. Z. Li, and L. Schmidt. Sample-optimal density estimation in nearly-linear time. CoRR, abs/1506.00671, 2015. 3.2

[2] N. Ailon, B. Chazelle, S. Comandur, and D. Liu. Property-preserving data reconstruction. Algorithmica, 51(2):160-182, 2008. 1.4

[3] V. Barnett. The study of outliers: purpose and model. Applied Statistics, pages 242-250, 1978. 1

[4] T. Batu, S. Dasgupta, R. Kumar, and R. Rubinfeld. The complexity of approximating the entropy. SIAM Journal on Computing, 35(1):132-150, 2005. 1.2

[5] T. Batu, L. Fortnow, R. Rubinfeld, W. D. Smith, and P. White. Testing that distributions are close. In Proceedings of FOCS, pages 189-197, 2000. 6

[6] T. Batu, L. Fortnow, R. Rubinfeld, W. D. Smith, and P. White. Testing closeness of discrete distributions. ArXiV, abs/1009.5397, 2010. This is a long version of [5]. 1.2

[7] T. Batu, R. Kumar, and R. Rubinfeld. Sublinear algorithms for testing monotone and unimodal distributions. In Proceedings of STOC, pages 381-390, New York, NY, USA, 2004. ACM. 1.2

[8] A. Bhattacharyya, E. Grigorescu, M. Jha, K. Jung, S. Raskhodnikova, and D. P. Woodruff. Lower bounds for local monotonicity reconstruction from 
transitive-closure spanners. SIAM Journal on Discrete Mathematics, 26(2):618-646, 2012. 1.4

[9] L. Birgé. On the risk of histograms for estimating decreasing densities. The Annals of Statistics, 15(3):pp. 1013-1022, 1987. 1.2, 1.2, 1.2

[10] M. Blum, M. Luby, and R. Rubinfeld. Self-testing/correcting with applications to numerical problems. In Proceedings of the Twenty-second Annual ACM Symposium on Theory of Computing, STOC '90, pages 73-83, New York, NY, USA, 1990. ACM. 1.4

[11] Z. Brakerski. Local property restoring. Manuscript, 2008. 3.3

[12] A. Campagna, A. Guo, and R. Rubinfeld. Local reconstructors and tolerant testers for connectivity and diameter. CoRR, abs/1208.2956, 2012. 3.3

[13] C. L. Canonne, I. Diakonikolas, T. Gouleakis, and R. Rubinfeld. Testing Shape Restrictions of Discrete Distributions. ArXiV, abs/1507.03558, July 2015. 1.2

[14] C. L. Canonne, D. Ron, and R. A. Servedio. Testing equivalence between distributions using conditional samples. In Proceedings of SODA, pages 1174-1192. Society for Industrial and Applied Mathematics (SIAM), 2014. 1.3

[15] C. L. Canonne and R. Rubinfeld. Testing probability distributions underlying aggregated data. In Proceedings of ICALP, pages 283-295, 2014. 1.2, 1.3

[16] S. Chakraborty, E. Fischer, Y. Goldhirsh, and A. Matsliah. On the power of conditional samples in distribution testing. In Proceedings of the 4th conference on Innovations in Theoretical Computer Science, ITCS '13, pages 561-580, New York, NY, USA, 2013. ACM. 1.3

[17] S.-O. Chan, I. Diakonikolas, R. A. Servedio, and $\mathrm{X}$. Sun. Learning mixtures of structured distributions over discrete domains, chapter 100, pages 1380-1394. SIAM, 2013. 1.2, 3.1

[18] S.-O. Chan, I. Diakonikolas, R. A. Servedio, and X. Sun. Efficient density estimation via piecewise polynomial approximation. In Proceedings of the 46th Annual ACM Symposium on Theory of Computing, STOC '14, pages 604-613, New York, NY, USA, 2014. ACM. 1.2, 3.2

[19] C. Daskalakis, I. Diakonikolas, R. O'Donnell, R. A. Servedio, and L.-Y. Tan. Learning sums of independent integer random variables. In Proceedings of the 2013 IEEE 54th Annual Symposium on Foundations of Computer Science, FOCS '13, pages 217-226, Washington, DC, USA, 2013. IEEE Computer Society. 1.2

[20] C. Daskalakis, I. Diakonikolas, and R. A. Servedio. Learning Poisson Binomial Distributions. In Proceedings of the Forty-fourth Annual ACM Symposium on Theory of Computing, STOC '12, pages 709-728, New York, NY, USA, 2012. ACM. 1.2, 3.2, 3.2

[21] C. Daskalakis, I. Diakonikolas, and R. A. Servedio. Learning $k$-modal distributions via testing. Theory of Computing, 10(20):535-570, 2014. 1.2, 1.2, 3.3, 4.1

[22] C. Daskalakis, I. Diakonikolas, R. A. Servedio, G. Valiant, and P. Valiant. Testing $k$-modal distributions: Optimal algorithms via reductions. In Proceedings of SODA, pages 1833-1852. Society for
Industrial and Applied Mathematics (SIAM), 2013. 1.2, 3.6

[23] C. Daskalakis and G. Kamath. Faster and sample near-optimal algorithms for proper learning mixtures of Gaussians. In Proceedings of The 27th Conference on Learning Theory, Barcelona, Spain, June 13-15, 2014, COLT '14, pages 1183-1213, 2014. 1.3

[24] A. P. Dempster, N. M. Laird, and D. B. Rubin. Maximum likelihood from incomplete data via the em algorithm. JOURNAL OF THE ROYAL STATISTICAL SOCIETY, SERIES B, 39(1):1-38, 1977. 1.4

[25] O. Goldreich and D. Ron. On testing expansion in bounded-degree graphs. Technical Report TR00-020, Electronic Colloquium on Computational Complexity (ECCC), 2000. 1.2

[26] U. Grenander. On the theory of mortality measurement. Scandinavian Actuarial Journal, 1956(1):70-96, 1956. 1.2

[27] D. M. Hawkins. Identification of outliers, volume 11. Springer, 1980. 1

[28] T. J. Hefley, D. M. Baasch, A. J. Tyre, and E. E. Blankenship. Correction of location errors for presence-only species distribution models. Methods in Ecology and Evolution, 5(3):207-214, 2014. 1

[29] B. Iglewicz and D. C. Hoaglin. How to detect and handle outliers, volume 16. Asq Press, 1993. 1

[30] R. Impagliazzo and D. Zuckerman. How to recycle random bits. In Proceedings of the 30th Annual Symposium on Foundations of Computer Science, SFCS '89, pages 248-253, Washington, DC, USA, 1989. IEEE Computer Society. 4

[31] P. Indyk, R. Levi, and R. Rubinfeld. Approximating and testing k-histogram distributions in sub-linear time. In Proceedings of the 31st Symposium on Principles of Database Systems, PODS '12, pages 15-22, New York, NY, USA, 2012. ACM. 1.2

[32] M. Jha and S. Raskhodnikova. Testing and reconstruction of Lipschitz functions with applications to data privacy. In Proceedings of FOCS, pages 433-442, Oct 2011. 1.4

[33] E. Kushilevitz and A. Rosén. A randomness-rounds tradeoff in private computation. In Y. Desmedt, editor, Advances in Cryptology - CRYPTO '94, volume 839 of Lecture Notes in Computer Science, pages 397-410. Springer Berlin Heidelberg, 1994. 4

[34] R. J. A. Little and D. B. Rubin. Statistical Analysis with Missing Data. Wiley Series in Probability and Statistics. John Wiley \& Sons, 2002. Second edition. 1, 1.4

[35] L. Paninski. A coincidence-based test for uniformity given very sparsely sampled discrete data. IEEE Transactions on Information Theory, 54(10):4750-4755, 2008. 1.2

[36] S. Panzeri, C. Magri, and L. Carraro. Sampling bias. Scholarpedia, 3(9):4258, 2008. revision \#91742. 1

[37] D. B. Rubin. Multiple imputation for nonresponse in surveys. John Wiley \& Sons, 1987. 1.4

[38] M. Saar-Tsechansky and F. Provost. Handling missing values when applying classification models. J. Mach. Learn. Res., 8:1623-1657, Dec. 2007. 1 
[39] A. Sahai and S. Vadhan. Manipulating statistical difference. In DIMACS Series in Discrete Mathematics and Theoretical Computer Science, pages 251-270. American Mathematical Society, 1998. 1.1

[40] M. Saks and C. Seshadhri. Local monotonicity reconstruction. SIAM Journal on Computing, 39(7):2897-2926, 2010. 1.4

[41] J. L. Schafer. Analysis of incomplete multivariate data. CRC press, 1997. 1, 1.4

[42] P. D. Senese and J. A. Vasquez. A unified explanation of territorial conflict: Testing the impact of sampling bias, 1919-1992. International Studies Quarterly, 47(2):275-298, 2003. 1

[43] P. W. Signor and J. H. Lipps. Sampling bias, gradual extinction patterns and catastrophes in the fossil record. Geological Society of America Special Papers, 190:291-296, 1982. 1
[44] G. Valiant and P. Valiant. A CLT and tight lower bounds for estimating entropy. Electronic Colloquium on Computational Complexity (ECCC), 17:179, 2010. 46

[45] G. Valiant and P. Valiant. Estimating the unseen: A sublinear-sample canonical estimator of distributions. Electronic Colloquium on Computational Complexity (ECCC), 17:180, 2010. 46

[46] G. Valiant and P. Valiant. The power of linear estimators. In Proceedings of FOCS, pages 403-412, Oct. 2011. See also [44] and [45]. 1.2, 3.6

[47] P. Valiant. Private communication, May 2015. 1.2

[48] S. Yekhanin. Locally decodable codes. Now Publishers Inc., 2010. 1.4 